\title{
Synthesis and Optical Manifestation of NiO-Silica Nanocomposite
}

\author{
S. Chakrabarty and K. Chatterjee \\ Department of Physics and Technophysics, Vidyasagar University, Midnapore 721102, India \\ Correspondence should be addressed to K. Chatterjee, kuntal@mail.vidyasagar.ac.in
}

Received 17 March 2011; Accepted 4 May 2011

Academic Editors: G. A. Kachurin and D. Tsoukalas

Copyright ( $) 2011$ S. Chakrabarty and K. Chatterjee. This is an open access article distributed under the Creative Commons Attribution License, which permits unrestricted use, distribution, and reproduction in any medium, provided the original work is properly cited.

\begin{abstract}
$\mathrm{NiO}$-silica nanocomposites with average diameter ranging from $2-40 \mathrm{~nm}$ were prepared by sol-gel method followed by the heat treatment varying from $400^{\circ} \mathrm{C}$ to $1000^{\circ} \mathrm{C}$. The details of morphology and crystalline nature of all the as prepared samples were characterized by TEM, HRTEM, and XRD analysis. The planes obtained from SAED pattern supports the planes originated from XRD study. From the optical absorption study, it is revealed that the band gap energy of $\mathrm{NiO}$ can extensively be manipulated by composite formation with silica and the size variation of that nanocomposite. Absorption peak position varies almost linearly with the oxidation temperature of the samples. Photoluminescence spectroscopy reveals that NiO-silica nanocomposite, prepared at $600{ }^{\circ} \mathrm{C}$ and below, shows strong emission at $3.62 \mathrm{eV}$, but the nanocomposites with bigger size greatly hinder the effect of selective emissivity.
\end{abstract}

\section{Introduction}

In recent years, nanomaterials especially nanostructured metal oxides attract extensive interests due to their novel optical, electronic, magnetic, thermal, and mechanical properties [1]. NiO (nickelous oxide) nanoparticles are one such momentous metal oxide that tend to be P-type semiconductor with a wide band gap [2]. It has tremendous application in science and technology. It can be used as a transparent Ptype semiconducting layer $[3,4]$ and as an antiferro magnetic film [5] and can also be extensively used in smart windows [6], electrochemical supercapacitor [7-9], and dye-sensitized photocathode [10]. It exhibits anodic electrochromism. Due to excellent durability and electrochemical stability, low material cost as an ion storage material, large-spin optical density, and various manufacturing possibilities, $\mathrm{NiO}$ semiconductors become interesting topics for scientists.

Different methods have already been reported for the synthesis of $\mathrm{NiO}$ nanoparticles such as evaporation $[11,12]$, magnetron sputtering [13-15], and sol-gel [16]. Particularly, the optical behavior of $\mathrm{NiO}$ under different circumstances is becoming one of the prime searches of the scientists. Proffessor Guerra et al. [17] investigated the cathodoluminescence and photoluminescence of $\mathrm{NiO}$ where as Proffessor Kuzmin and his group [18] examined the effect of doping in the origin of visible photoluminescence in $\mathrm{NiO}$. Even the $\mathrm{NiO}$ nanowires are also under observation for their emission property [19]. However, the complexity remains in the synthesis technique as well as in the limitation of tailoring the optical properties of $\mathrm{NiO}$ nanoparticle. Especially the optical response of bare $\mathrm{NiO}$ nanoparticles cannot be tailored much for their large band-gap energy. Keeping this in mind, scientists have proposed to oxidize $\mathrm{Ni}$ nanoparticle within insulating medium by ion implantation method in search of better stability and novel optical properties [20]. Effort has been also delivered to synthesize $\mathrm{NiO}$ nanoparticle within insulating silica matrix to form nanocomposite films leading toward optical gas sensor $[21,22]$. In this paper Proffessor Martucci et al. have shown how the doping of $\mathrm{NiO}$ in $\mathrm{SiO}_{2}$ matrix, prepared by sol-gel route, can improve the sensing properties of the materials. But other different aspects of these potential NiO-silica nanocomposite materials still remain unearthed. In this work we concentrate on the synthesis and optical characterization of differently sized $\mathrm{NiO}$-silica nanocomposites prepared by sol-gel technique to tailor the optical band gap in a different approach from the route adopted by ProffessorMartucci et al. [21].

Here well-dispersed Ni nanoparticles were prepared first within $\mathrm{SiO}_{2}$ matrix by sol-gel method and then by a controlled heat treatment at elevated temperature ranging from $400^{\circ} \mathrm{C}$ to $1000^{\circ} \mathrm{C}$, the $\mathrm{NiO}$-silica nanocomposites were prepared with diameter varying from 2 to $40 \mathrm{~nm}$. 
The crystalline phases of all as-prepared $\mathrm{NiO}$-silica samples were identified by X-ray diffraction (XRD). The structure and morphological properties were studied by TEM and HRTEM. The variation in optical absorbance of different $\mathrm{NiO}$-silica nanocomposites was examined thoroughly. The detail of PL study of the different-sized NiO-silica nanocomposites reveal interesting emission peak at around $3.6 \mathrm{eV}$ for the sample prepared with the heat treatment below $700^{\circ} \mathrm{C}$ but for NiO-silica composites synthesized at higher temperature $\left(\geq 700^{\circ} \mathrm{C}\right)$ do not exhibit any emission property. The result was discussed on the basis of the electronic transition of the $3 \mathrm{~d}$ state of the $\mathrm{Ni}^{2+}$ ion and the surface defect states.

\section{Experiment}

Firstly, a fresh solution was made by taking $0.06 \mathrm{M} \mathrm{NiCl}_{2}$, $50 \mathrm{~mL} \mathrm{C}_{2} \mathrm{H}_{5} \mathrm{OH}$, and $5 \mathrm{~mL}$ distilled water and the prepared solution, placed in ice bath, was stirred by a magnetic stirrer for $1 \mathrm{~h}$. This solution was named as "A". In another beaker $0.05 \mathrm{M}$ TEOS, $\mathrm{C}_{2} \mathrm{H}_{5} \mathrm{OH}$, and few drops of diluted $\mathrm{HCl}$ were taken, and the colorless solution of $\mathrm{pH} 6$ was stirred for $1 \mathrm{~h}$ keeping the system in ice bath. This solution was named as "B". The solution A was then added to the solution B and stirred for few minutes. One transparent green solution was prepared as "Sol", and it was kept in a Petridis for few days for gelation. After the complete "Gel" formation, the sample was crashed to powder, and a solution was prepared dispersing the powdered sample into distilled water. The solution was placed in the ice bath and stirred for 20 minutes and was kept as "C". Another saturated solution of $\mathrm{NaBH}_{4}$ with distilled water was prepared and was kept as "D". Then the solution " $\mathrm{D}$ " was added to solution " $\mathrm{C}$ " very quickly resulting in a black precipitation. Next, the solution was stirred for 10 minutes, and then the products were collected by centrifugation. The dried sample was treated at different oxidation temperature ranging from $400^{\circ} \mathrm{C}-1000^{\circ} \mathrm{C}$ for 10 minutes to prepare $\mathrm{NiO}$-silica nanocomposites of various sizes.

All the powdered samples were characterized both by Rigaku Mini flex X-Ray diffractometer using $\mathrm{Cu} \mathrm{K}_{\alpha}$ radiation source as well as by JEM 2010 transmission electron microscope. Optical absorption spectra of the powdered samples were recorded in a UV-VIS 1700 Shimadzu Spectrophotometer. The powdered sample were dispersed in ethyl alcohol and mounted in the sample chamber while pure ethyl alcohol was taken in the reference beam position. For photoluminescence measurement, the samples were also taken in ethyl alcohol, and the measurements were carried out in LS55 (Perkin Elmer) PL Spectrophotometer.

\section{Result and Discussion}

Figure 1 shows the details of the XRD patterns of the $\mathrm{NiO}$-silica nanocomposites obtained at different oxidation temperatures. It is evident from the figure that the sample prepared at $400^{\circ} \mathrm{C}$ and $500^{\circ} \mathrm{C}$ has no appreciable peak, but as the oxidation temperature increases, the peaks are generated from different planes of $\mathrm{NiO}$ and silica. As the sample treatment temperature increases, the crystal size increases, and in the XRD pattern, the peak intensity and

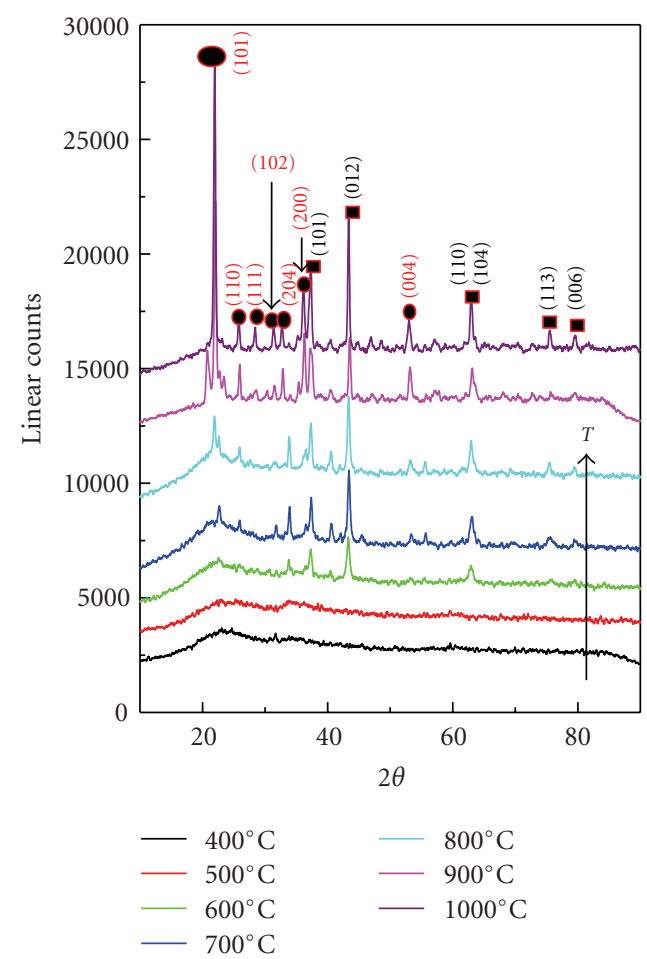

FIGURE 1: XRD pattern of NiO-silica nanocomposites prepared at different temperatures. represents $\mathrm{SiO}_{2}$ peak, and represents $\mathrm{NiO}$ peak.

sharpness increase accordingly. Here at comparatively low oxidation temperature, the silica is still in glass form, and as the temperature increases, it starts to become much more crystalline nucleating from the preexisting $\mathrm{Ni}$ site. Thus, with the increasing temperature the $\mathrm{NiO}$-silica composite form gets much stronger impression in the XRD data. The measurement also shows that annealing temperature of the different as-prepared samples did not alter their chemical phase in NiO. However, the relative intensities of different planes, indexed in the figure, were greatly improved by the elevated temperature, indicating that nanocrystals could grow with preferential orientation. It is worthy to notice that the crystalline phase of silica comes in the temperature well below the crystallization temperature $\left(1200^{\circ} \mathrm{C}\right)$ of pure bulk silica prepared by sol-gel technique. It is established that the crystallization behavior of $\mathrm{SiO}_{2}$ is strongly influenced by the ion species located in the circumference, and the crystallization temperature varies in a complicated manner depending on the methods of processing [23]. Here it is assumed that in presence of $\mathrm{Ni}$ site, the effective crystallization temperature of silica has been reduced substantially. It is obvious from the figure that the crystalline peak from silica starts to appear from $700^{\circ} \mathrm{C}$ onwards. The reason why the crystal planes of $\mathrm{NiO}$ were not observed in the XRD patterns of sample obtained from $400^{\circ} \mathrm{C}$ and $500^{\circ} \mathrm{C}$ heat treatment is not very clear. It is assumed that the peak intensity originated from the very small dimension of $\mathrm{NiO}$ nanocrystal within silica matrix is not sufficient for the detection unit of the instrument. It is known that the very small size of the crystal may not allow detecting the crystal planes by XRD study [21]. 


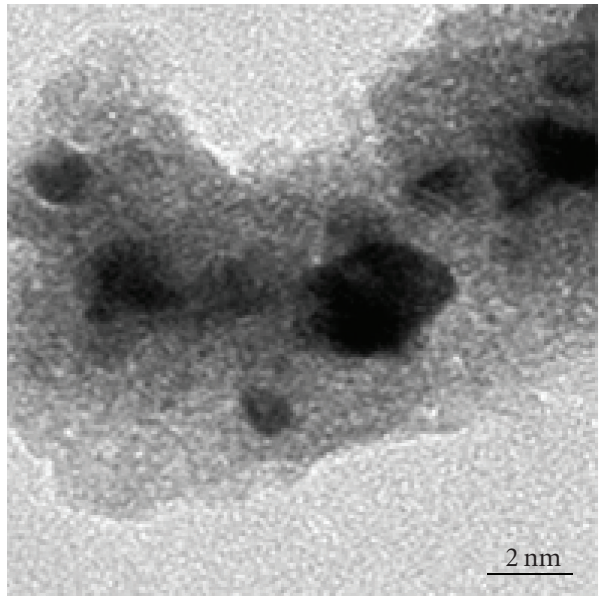

(a)

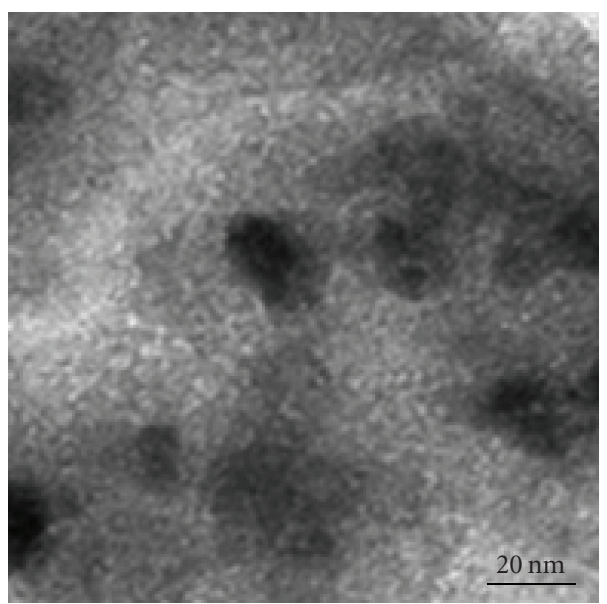

(c)

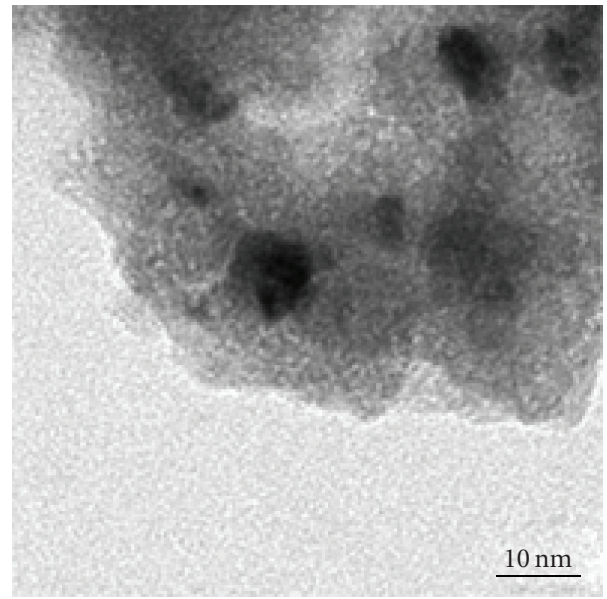

(b)

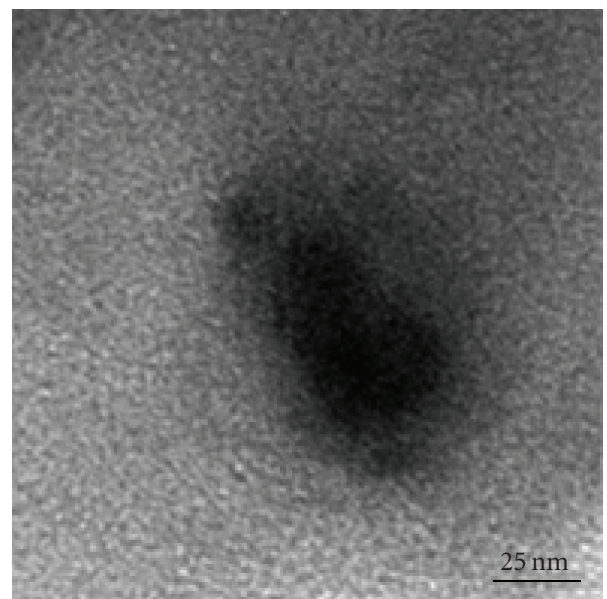

(d)

Figure 2: Typical TEM images of NiO-silica nanocomposites prepared at $400^{\circ} \mathrm{C}$ (a), at $600^{\circ} \mathrm{C}(\mathrm{b})$, at $800^{\circ} \mathrm{C}(\mathrm{c})$, and at $1000^{\circ} \mathrm{C}(\mathrm{d})$.

Figure 2 shows the typical TEM images of well-dispersed $\mathrm{NiO}$-silica nanocomposites obtained from four different oxidation temperatures. It is clearly seen that the particle size increases with the increasing oxidation temperature. Here for the sake of clarity, TEM image of all the heat-treated samples were not included in the figure, but typical four different type samples had been chosen. The average size of the synthesized nanocomposite from different oxidation treatment varies from $2 \mathrm{~nm}$ to $40 \mathrm{~nm}$. The smallest size arises due to $400^{\circ} \mathrm{C}$ heat treatment [Figure 2(a)], and the largest composite size comes due to the $1000^{\circ} \mathrm{C}$ heat treatment [Figure 2(d)]. The intermediate heat treatments give rise to the intermediate average particle sizes. Here it is interesting to note in the TEM image that nanocomposite prepared even at the lowest heat treatment $\left(400^{\circ} \mathrm{C}\right)$ has particle nature which confirms crystalline structure of the sample though it was not observed from the XRD study.

Figure 3(a) shows the selected-area electron diffraction pattern (SAED) originated from the NiO-silica nanocomposite taking one typical sample prepared at $800^{\circ} \mathrm{C}$, and the planes calculated from this diffraction rings are in well agreement with the planes evolved from our XRD study. Investigation of the different ring originated from the SAED pattern also supports the presence of both phases, $\mathrm{NiO}$ and $\mathrm{SiO}_{2}$, within the prepared sample. Figure 3(b) shows HRTEM of single typical NiO-silica nanocomposite confirming the well-crystalline phase of the sample. Here $d$ value calculated from the fringe pattern matches with the (101) planes of NiO signifying that the $\mathrm{NiO}$ phase is the dominating part of the composite particle which is likely to be. From this HRTEM it is also clear that prepared sample has structurally uniform $\mathrm{NiO}$ phase which grows in a single direction.

Optical absorption spectra of the different NiO-silica nanocomposites have been shown in Figure 4. From the figure, it is noticed that with the increasing oxidation temperature, the absorbance peak position of the samples is shifting towards higher wavelength. It is obvious that with higher oxidation temperature the crystal size increases, and we know that the optical absorbance peak get redshifted with the higher particle size. This absorbance, we assume, is not due to the band- to band-transition of NiO. 


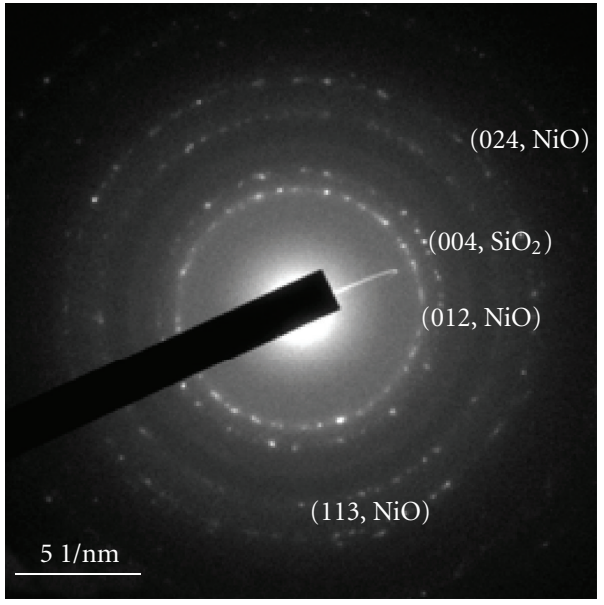

(a)

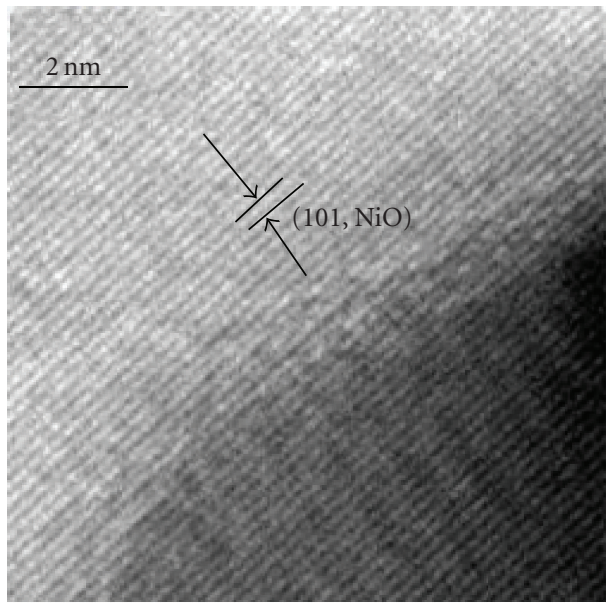

(b)

FIgURE 3: Typical (a) SAED image and (b) HRTEM of NiO-silica nanocomposite sample prepared at $800^{\circ} \mathrm{C}$. The corresponding planes are indexed in (a) and (b).

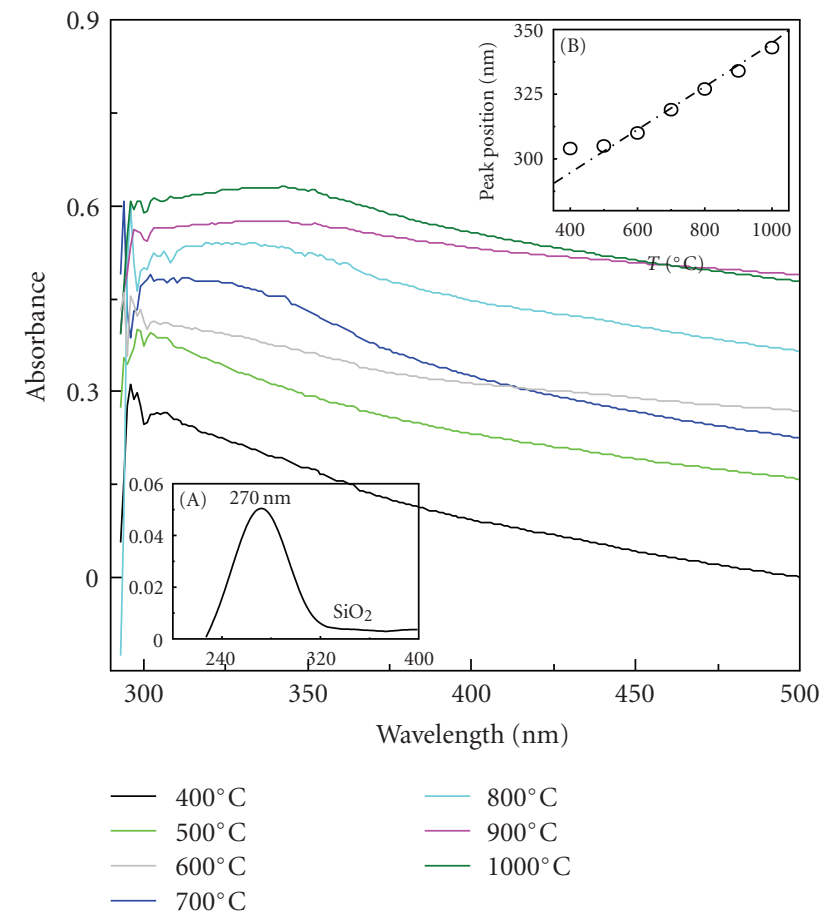

FIGURE 4: Optical absorption spectra for NiO-silica nanocomposite prepared at different temperatures. The inset A shows the optical absorbance of pure silica prepared by sol-gel method followed by $800^{\circ} \mathrm{C}$ heat treatment for $10 \mathrm{~min}$. Inset $\mathrm{B}$ shows absorbance peak position versus oxidation temperature curve of the $\mathrm{NiO}$-silica nanocomposite. Dotted line is for the eye guidance.

Rather due to the composite formation of $\mathrm{NiO}$ and $\mathrm{SiO}_{2}$, there may be some surface states in between the band, and the transition may occur from intermediate surface states to the conduction band providing a room to tailor the band-gap energy of the prepared $\mathrm{NiO}-\mathrm{SiO}_{2}$ nanocomposite. Pure silica prepared by sol-gel method followed by heat treatment for 10 minutes at $400^{\circ} \mathrm{C}$ to $1000^{\circ} \mathrm{C}$ with $100^{\circ} \mathrm{C}$ interval gives the absorption peak at $270 \mathrm{~nm}$ which is far away from the peak position originated from the prepared nanocomposites. One such absorption behavior of silica sample heated at $800^{\circ} \mathrm{C}$ is shown in the inset A of Figure 4 to ensure that the absorptions coming from the samples are not the contribution of pure silica.

It is seen from the figure that the peak position of the $\mathrm{NiO}$-silica nanocomposite varies in the UV region from $300 \mathrm{~nm}$ to $340 \mathrm{~nm}$, and the variation of absorbance peak position with the temperature is almost in linear relation shown in the inset B of Figure 4. Here a dotted line is drawn for the eye guidance. This simple linear behavior provides the splendid opportunity to tailor the size of $\mathrm{NiO}$-silica nanocomposites to tune the absorption peak position.

Figure 5 shows the photoluminescence response curves taken from three different $\mathrm{NiO}$-silica samples prepared at $400^{\circ} \mathrm{C}, 500^{\circ} \mathrm{C}$, and $600^{\circ} \mathrm{C}$. All these samples show one strong peak at $3.62 \mathrm{eV}(341 \mathrm{~nm})$ and two shoulders on both side of the main peak centered at about $3.75 \mathrm{eV}(330 \mathrm{~nm})$ and $3.46 \mathrm{eV}(357 \mathrm{~nm})$. Interestingly, the nanocomposites prepared at higher temperature, $700^{\circ} \mathrm{C}$ onwards, are not showing any distinct emission peak at those energy levels, and for that reason, they are not shown in the figure. Here for the sake of comparison, photoluminescence responses originating from the pure silica, prepared by sol-gel method and heat treated at respective temperature, are shown as well. From this experiment, it is clear that the feeble shoulder originating at $330 \mathrm{~nm}$ in the nanocomposite sample is originally attributed to the silica phase, and the main peak is originating from the electronic transition of the $\mathrm{Ni}^{2+}$ ions. In $\mathrm{NiO}$ the existence of several transitions at energies below band gap has been reported by different scientists using optical absorption study $[18,24]$. Adler and Feinleib [25] reported a series of absorption peaks below $4 \mathrm{eV}$ as purely intraionic $3 \mathrm{~d}^{8}-3 \mathrm{~d}^{8}$ transitions of $\mathrm{Ni}^{2+}$. By the study of electron energy loss spectroscopy (EELS) and spin-polarized 


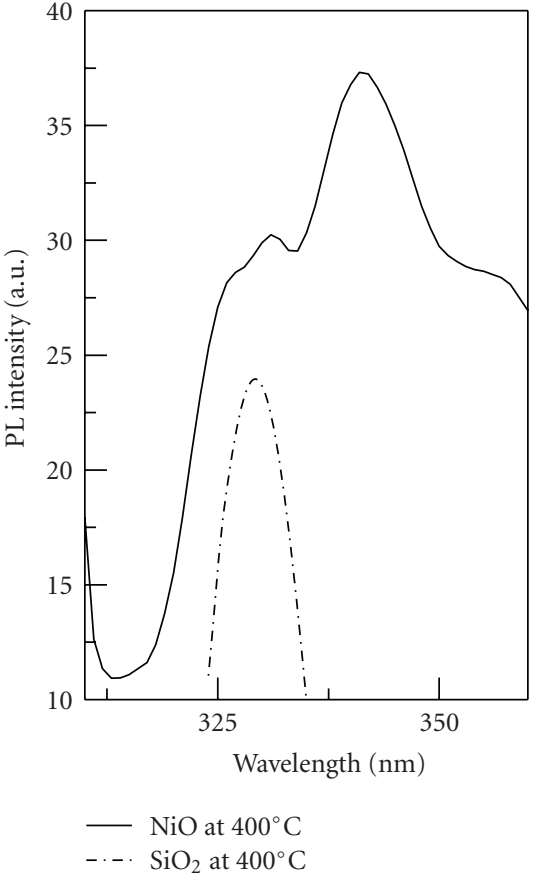

(a)

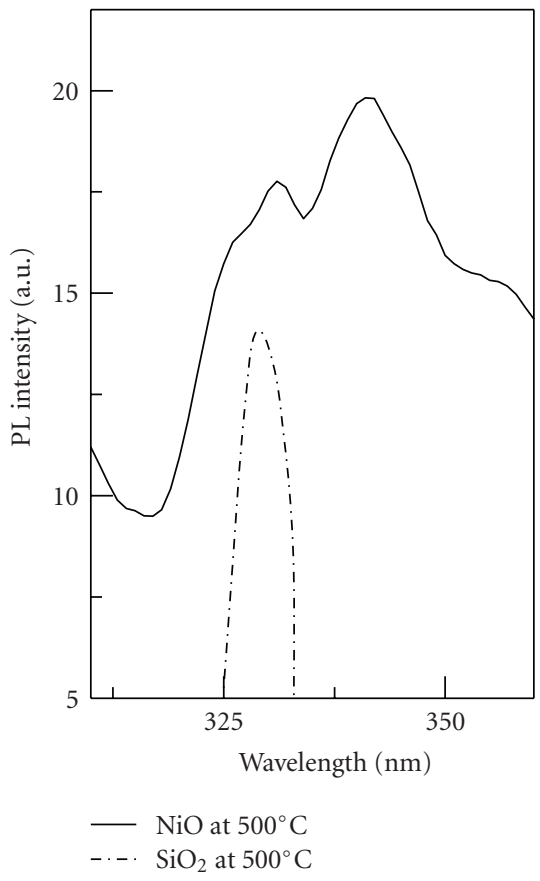

(b)

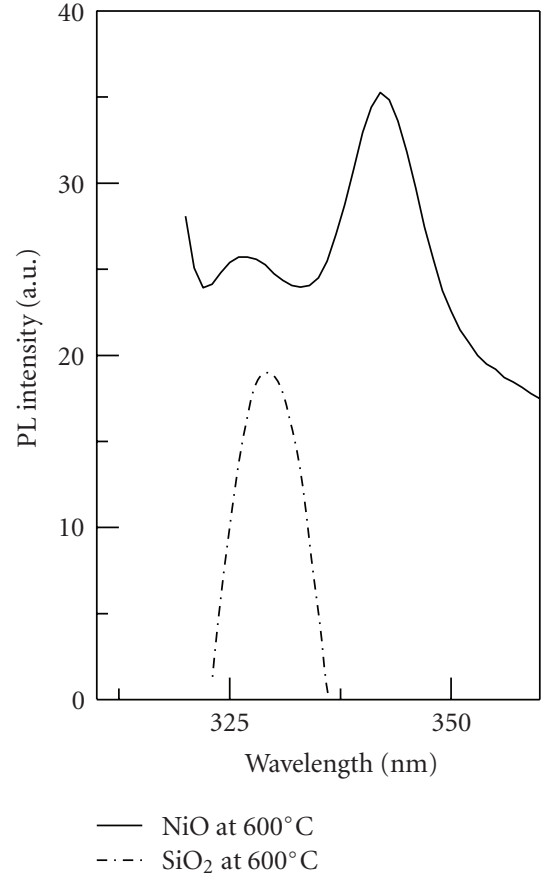

(c)

Figure 5: PL spectra of NiO-silica nanocomposite prepared at three different temperatures (a)-(c), (a. u. means arbitrary units). PL spectra of pure silica prepared at the same temperature are shown by the dotted line for each sample. All the samples were excited at $4.12 \mathrm{eV}(300 \mathrm{~nm})$, and the data are taken in RT.

electron energy loss spectroscopy (SPEELS), the existence of this kind of transition of the $3 \mathrm{~d}^{8}$ electrons of $\mathrm{Ni}^{2+}$ was also confirmed $[26,27]$. But the interesting thing is that the samples prepared at and above $700^{\circ} \mathrm{C}$ are not showing this emission band at all. One can notice from Figure 1 that, for the samples prepared at $700^{\circ} \mathrm{C}$ onwards, the existence of silica phase becomes stronger. So, it can be assumed that for the sample prepared at the above-said temperatures the number of surface states is so much that they do not allow the radiative relaxation. It is also reported that NiOs are very much susceptible to the heat treatment for their stoichiometric change in behavior [19]. In the lower oxidation temperature, the particle size and the absence of strongsilica phase provides the opportunity of selective relaxation emission whereas in the higher temperature treatment the silica phase and the effective size of the nanocomposites suppress the quality of emission from the $\mathrm{NiO}$.

\section{Conclusions}

In summery $\mathrm{NiO}$-silica nanocomposites were synthesized by very-easy-to-achieve chemical synthesis route. The size of the nanocomposite was varied from 2 to $40 \mathrm{~nm}$ by controlling the oxidation temperature. All the samples were characterized by XRD, TEM, and HRTEM analysis. Optical absorbance study reveals that absorbance peak position varies from $300 \mathrm{~nm}$ to $340 \mathrm{~nm}$ as the particle size increases. Moreover, photoluminescence investigation reveals that the $\mathrm{NiO}$-silica systems before the crystallization of silica show strong emission peak at around $3.62 \mathrm{eV}$ while nanocompos- ites prepared at higher oxidation temperature $\left(\geq 700^{\circ} \mathrm{C}\right)$ with the strong crystalline phase of silica do not show emission peaks at all.

\section{Acknowledgment}

The authors gratefully acknowledge the financial support from FIST, Department of Science and Technology, India and SAP, University Grant Commission, India.

\section{References}

[1] J. L. Gunjakar, A. M. More, and C. D. Lokhande, "Chemical deposition of nanocrystalline nickel oxide from urea containing bath and its use in liquefied petroleum gas sensor," Sensors and Actuators B, vol. 131, no. 2, pp. 356-361, 2008.

[2] B. Subramanian, M. M. Ibrahim, V. Senthilkumar et al., "Optoelectronic and electrochemical properties of nickel oxide (NiO) films deposited by DC reactive magnetronsputtering," Physica B, vol. 403, no. 21-22, pp. 4104-4110, 2008.

[3] X. Wang, J. Song, L. Gao, J. Jin, H. Zheng, and Z. Zhang, "Optical and electrochemical properties of nanosized $\mathrm{NiO}$ via thermal decomposition of nickel oxalate nanofibres," Nanotechnology, vol. 16, no. 1, pp. 37-39, 2005.

[4] B. Sasi, K. G. Gopchandran, P. K. Manoj, P. Koshy, P. P. Rao, and V. K. Vaidyan, "Preparation of transparent and semiconducting NiO films," Vacuum, vol. 68, no. 2, pp. 149-154, 2002.

[5] S. R. Krishnakumar, M. Liberati, C. Grazioli et al., "Magnetic linear dichroism studies of in situ grown NiO thin films," Journal of Magnetism and Magnetic Materials, vol. 310, no. 1, pp. 8-12, 2007. 
[6] C. G. Granqvist, Handbook of Electrochromic Materials, Elsevier, Amsterdam, The Netherlands, 1995.

[7] X. Wang, L. Li, Y. Zhang et al., "High-yield synthesis of $\mathrm{NiO}$ nanoplatelets and their excellent electrochemical performance," Crystal Growth and Design, vol. 6, no. 9, pp. 2163-2165, 2006.

[8] T. Nathan, A. Aziz, A. F. Noor, and S. R. S. Prabaharan, "Nanostructured $\mathrm{NiO}$ for electrochemical capacitors: synthesis and electrochemical properties," Journal of Solid State Electrochemistry, vol. 12, no. 7-8, pp. 1003-1009, 2008.

[9] M. Wu, J. Gao, S. Zhang, and A. Chen, "Comparative studies of nickel oxide films on different substrates for electrochemical supercapacitors," Journal of Power Sources, vol. 159, pp. 365-369, 2006.

[10] L. Lin, E. A. Gibson, P. Qin et al., "Double-layered NiO photocathodes for p-type DSSCs with record IPCE," Advanced Materials, vol. 22, no. 15, pp. 1759-1762, 2010.

[11] K. Tetsuo and Y. Toshikazu, "Preparation and structural analysis of nickel/nickel oxide multilayers," Journal of Applied Physics, vol. 77, no. 12, pp. 6651-6657, 1995.

[12] W. Wang, Y. Liu, C. Xu, C. Zheng, and G. Wang, "Synthesis of $\mathrm{NiO}$ nanorods by a novel simple precursor thermal decomposition approach," Chemical Physics Letters, vol. 362, no. 1-2, pp. 119-122, 2002.

[13] G. H. Yu, L. R. Zeng, F. W. Zhu, C. L. Chai, and W. Y. Lai, "Magnetic properties and X-ray photoelectron spectroscopy study of $\mathrm{NiO} / \mathrm{NiFe}$ films prepared by magnetron sputtering," Journal of Applied Physics, vol. 90, no. 8, pp. 4039-4043, 2001.

[14] S. Nandy, U. N. Maiti, C. K. Ghosh, and K. K. Chattopadhyay, "Enhanced p-type conductivity and band gap narrowing in heavily $\mathrm{Al}$ doped $\mathrm{NiO}$ thin films deposited by RF magnetron sputtering," Journal of Physics, vol. 21, no. 11, article 115804, 2009.

[15] H. Hahn and R. S. Averback, "The production of nanocrystalline powders by magnetron sputtering," Journal of Applied Physics, vol. 67, no. 2, pp. 1113-1115, 1990.

[16] L. Xiang, X. Y. Deng, and Y. Jin, "Experimental study on synthesis of NiO nano-particles," Scripta Materialia, vol. 47, no. 4, pp. 219-224, 2002.

[17] C. D. Guerra, A. Remon, J. A. Garcia, and J. Piqueras, "Cathodoluminescence and photoluminescence spectroscopy of NiO," Physica Status Solidi (a), vol. 163, no. 2, pp. 497-503, 1997.

[18] A. Kuzmin, N. Mironova-Ulmane, and S. Ronchin, "Origin of visible photoluminescence in $\mathrm{NiO}$ and $\mathrm{Ni}_{c} \mathrm{Mg}_{1-c} \mathrm{O}$ singlecrystals," Proceedings of SPIE, vol. 5122, pp. 61-67, 2003.

[19] H. Cao, X. Qiu, Y. Liang, L. Zhang, M. Zhao, and Q. Zhu, "Sol_-gel template synthesis and photoluminescence of nand p-type semiconductor oxide nanowires," ChemPhysChem, vol. 7, no. 2, pp. 497-501, 2006.

[20] H. Amekura, Y. Takeda, K. Kono, H. Kitazawa, and N. Kishimoto, "Modification of metal nanoparticles in $\mathrm{SiO} 2$ by thermal oxidation," Reviews on Advanced Materials Science, vol. 5, no. 3, pp. 178-182, 2003.

[21] A. Martucci, N. Bassiri, M. Guglielmi, L. Armelao, S. Gross, and J. C. Pivin, "NiO-SiO2 sol-gel nanocomposite films for optical gas sensor," Journal of Sol-Gel Science and Technology, vol. 26, no. 1-3, pp. 993-996, 2003.

[22] L. Zbroniec, A. Martucci, T. Sasaki, and N. Koshizaki, "Optical $\mathrm{CO}$ gas sensing using nanostructured $\mathrm{NiO}$ and $\mathrm{NiO} / \mathrm{SiO} 2$ nanocomposites fabricated by PLD and sol-gel methods," Applied Physics A, vol. 79, no. 4-6, pp. 1303-1305, 2004.
[23] Y. Kanno, "Discussion from the nucleation for the crystallization temperature of vitreous silica," Journal of Materials Science Letters, vol. 9, no. 4, pp. 451-453, 1990.

[24] T. Tsuboi and W. Kleemann, "Fine structure of near infrared optical absorption in NiO," Journal of Physics, vol. 6, no. 41, pp. 8625-8631, 1994.

[25] D. Adler and J. Feinleib, "Electrical and optical properties of narrow-band materials," Physical Review B, vol. 2, no. 8, pp. 3112-3134, 1970.

[26] A. Gorschluter and H. Merz, "Localized d-d excitations in $\mathrm{NiO}(100)$ and $\mathrm{CoO}(100)$," Physical Review B, vol. 49, no. 24, pp. 17293-17302, 1994.

[27] B. Fromme, M. Moller, TH. Anschutz, C. Bethke, and E. Kisker, "Electron-exchange processes in the excitations of $\mathrm{NiO}(100)$ surface d states," Physical Review Letters, vol. 77, no. 8, pp. 1548-1551, 1996. 

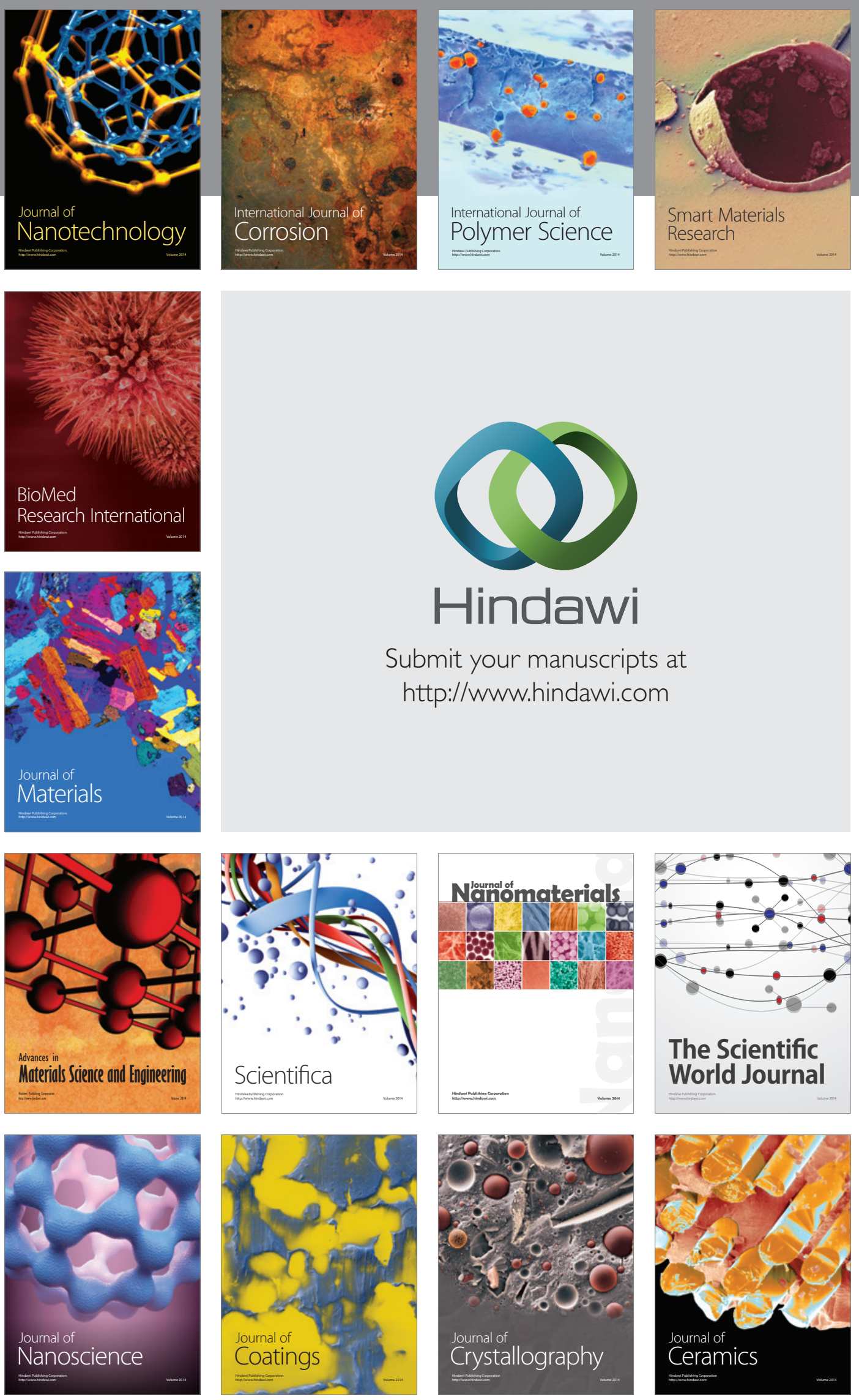

The Scientific World Journal

Submit your manuscripts at

http://www.hindawi.com

\section{World Journal}

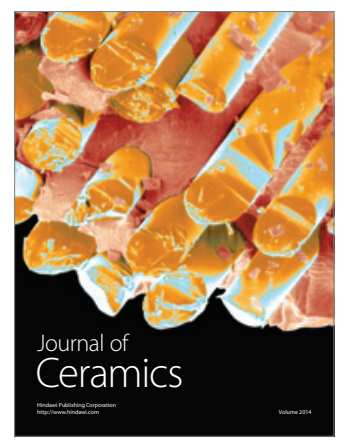

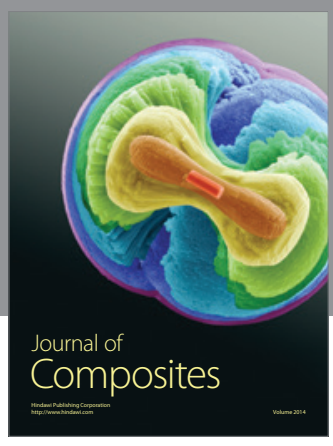
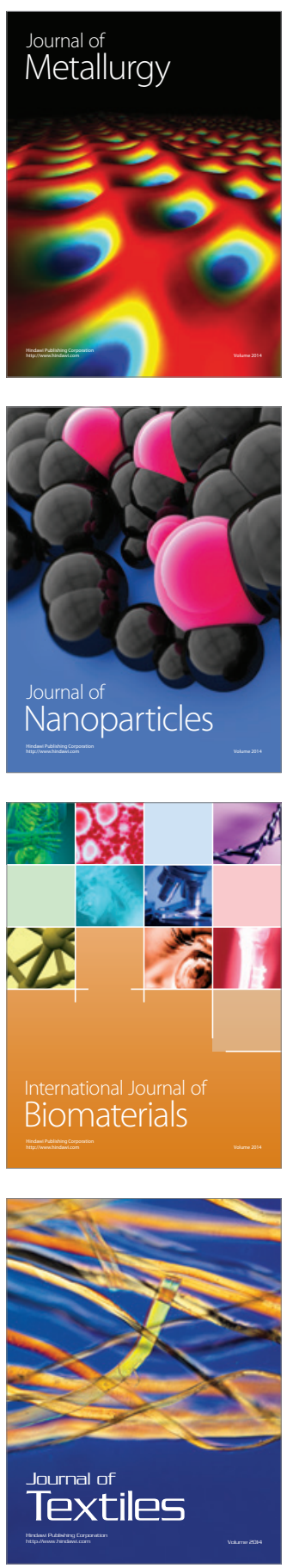